\title{
Diagnostic Accuracy of Breast MRI Compared with Conventional Imaging, in the Evaluation of Patients with Suspicious Nipple Discharge
}

\section{Giovanna Panzironi ${ }^{1 *}$, Francesca Galati' , Flaminia Marzocca ${ }^{1}$, Miles Kirchin ${ }^{2}$ and Federica Pediconi ${ }^{1}$}

${ }^{1}$ Department of Radiological Sciences, Sapienza University of Rome, Italy

${ }^{2}$ Bracco Imaging, Italy

\begin{abstract}
Purpose: To determine the role and the diagnostic accuracy of breast Magnetic Resonance Imaging in the evaluation of patients with suspicious discharge from the nipple.

Methods: 79 patients with suspicious nipple discharge underwent 3 Tesla MRI. Images were acquired with T2-IDEAL sequences, diffusion-weighted sequences as well as 3D T1-weighted sequences before and after the administration of $0.1 \mathrm{mmol} / \mathrm{kg}$ of gadobenate dimeglumine. MRI findings were classified according to Breast Imaging Reporting and Data System criteria as benign (BIRADS 1-2-3) or malignant (BIRADS 4-5). Reference diagnoses were based on histologic samples obtained at surgery for 51 patients and on clinical and instrumental follow-up over 24 months for 28 patients.

Results: MRI identified 32 cases of BIRADS 4-5, 27 of which were found to be malignant at histological examination. In the 47 cases classified as BIRADS 1-2-3, 17 lesions were confirmed as benign by definitive histological examination, 2 lesions were found to be a malignant tumor and 28 patients had negative follow-up at 24 months. Sensitivity, specificity and diagnostic accuracy of MRI were respectively $93.1 \%, 90 \%$ and $91.1 \%$ with a positive predictive value of $84.3 \%$ and negative predictive value of $95.7 \%$. The performance of MRI was also evaluated in comparison of positive cases of mammography and ultrasound.
\end{abstract}

Conclusion: Breast MRI is an accurate method in the evaluation of patients with suspicious nipple secretion. A negative MRI can direct patients to follow-up rather than surgery.

Keywords

Magnetic resonance imaging, Nipple discharge, Breast cancer, Breast imaging

\section{Introduction}

Nipple discharge (ND) is the third most common complaint involving the breast, after pain and pal- pable masses [1]. Whereas most cases of ND are hormonal or physiological in nature, deriving from multiple ducts and often bilateral [2-4], pathologi-

*Corresponding author: Giovanna Panzironi, MD, Department of Radiological Sciences, Sapienza University of Rome; Policlinico Umberto I, Viale Regina Elena 324, 00161 Rome, Italy

Accepted: September 18, 2018; Published: September 20, 2018

Copyright: (c) 2018 Panzironi G, et al. This is an open-access article distributed under the terms of the Creative Commons Attribution License, which permits unrestricted use, distribution, and reproduction in any medium, provided the original author and source are credited. 
cal nipple discharge (PND) is typically spontaneous, persistent and unilateral, deriving from a single duct during a non-lactational period [5]. In most cases of PND, the cause of the discharge is a benign lesion, with intraductal papilloma or papillomatosis the most frequent cause followed by duct ectasia and fibrocystic changes [5-10]. Unfortunately, in roughly $15-20 \%$ of cases the cause of PND is breast cancer, most commonly papillary carcinoma, ductal carcinoma in situ (DCIS), or invasive ductal carcinoma (IDC) [5-13].

Of the various approaches to non-invasively diagnosing the cause of PND, MR imaging (MRI) has proven more reliable and accurate than galactography for patient workup particularly when conventional mammography and ultrasound exams are negative or non-diagnostic [4,6-9,14-20]. MRI has demonstrated superior diagnostic performance both for the detection of otherwise occult disease and for its accurate characterization as benign or malignant.

Our study sought to further investigate the value of MRI in patients with suspicious ND with diagnostic performance determined using findings from histology or 2-year follow-up as reference standard.

\section{Materials and Methods}

\section{Patients}

Between February 2014 and February 2015, 79 consecutive women (mean age: 49.9 years; range 21-84 years) with unilateral bloody, serous or serous-bloody nipple discharge were included in this prospective single-centre study. All patients underwent breast MRI after an initial Ultrasound (US) exam. Patients older than 35 years also underwent conventional Digital Mammography (DM). DM imaging was acquired with a Full Field DM system (Siemens MAMMOMAT Inspiration; Siemens AG Healthcare, Erlangen, Germany). US evaluation was performed with a standard ultrasound system (Aplio 500; Toshiba, Canada Medical Systems) with high-resolution linear array 5-12 MHz transducer.

Patients with contraindications to MRI such as metallic foreign bodies or intolerance to gadolinium contrast agents were excluded. Local institutional review board approved the study protocol and written informed consent was obtained from all individual participants included in the study.

\section{MRI of the breast}

Breast MRI was performed on 3.0 Tesla magnet
(Discovery MR750, GE Medical Systems, Milwaukee, Wisconsin, USA) using an 8-channel bilateral breast surface coil with the patient in the prone position.

The 3.0 Tesla MR protocol included Fast Spin Echo T2 IDEAL (Iterative Decomposition of Water and Fat with Echo Asymmetry and Least Squares Estimation) sequences, Diffusion Weighted Imaging sequences and Vibrant (Volume Imaging for Breast Assessment) 3D T1-weighted sequences acquired before and after the intravenous administration of gadolinium contrast agent (gadobenate dimeglumine [MultiHance]; Bracco Imaging SpA, Milan, Italy). The T2-weighted IDEAL sequence was acquired with: TR/TE (Repetition time/Echo Time): $9128 / 120 \mathrm{~ms}$; flip angle: $30^{\circ}$; matrix: $320 \times 256$ pixels (pixel size $1.1 \times 1.3 \mathrm{~mm}$ ); field-of-view (FOV): $340 \mathrm{~mm}$; slice thickness: $3 \mathrm{~mm}$; acquisition time: $200 \mathrm{sec}$. The multiecho-single-shot EPI-DWI sequence was acquired with TR/TE: 6,8406,236/51 ms; matrix: $96 \times 128$ pixels; FOV: $360 \times 360$ $\mathrm{mm}$ (pixel size $3.6 \times 2.8 \mathrm{~mm}$ ); section thickness: 3 $\mathrm{mm}$; intersection gap: $0.3 \mathrm{~mm}$; b value: $0 \mathrm{~mm}^{2} / \mathrm{sec}$, $500 \mathrm{~mm}^{2} / \mathrm{sec}, 1000 \mathrm{~mm}^{2} / \mathrm{sec}$; acquisition time: 240 sec. The Vibrant fast 3D Fat Sat Spectral Saturation T1-weighted sequence was acquired with TR/TE: 7.7/4.2 ms; flip angle: $15^{\circ}$; matrix: $512 \times 256$ pixels; FOV: $350 \times 490 \mathrm{~mm}$ (pixel size $0.7 \times 1.5 \mathrm{~mm}$ ); slice thickness: $1.2 \mathrm{~mm}$; acquisition time: $7-8$ minutes, including one pre-contrast phase and 7 phases acquired after injection of gadobenate dimeglumine at a dose of $0.1 \mathrm{mmol} / \mathrm{kg}$ bodyweight.

Gadobenate dimeglumine was injected through an antecubital venous access with an automatic injector (Spectris, Medrad, Warrendale, Palo Alto, USA) at a rate of $2 \mathrm{~mL} / \mathrm{sec}$ and was followed by 20 $\mathrm{mL}$ of saline flush administered at the same rate.

All images were acquired in the axial plane. Imaging of premenopausal women was performed between the $7^{\text {th }}$ and $14^{\text {th }}$ day of the menstrual cycle.

\section{Image evaluation}

Original source images as well as subtracted images and Maximum Intensity Projection (MIP) reconstructions were evaluated in consensus by two experienced radiologists (with over 12 years and 3 years of experience in breast imaging) who were unaware of the results of initial clinical examinations, as well as the results of conventional imaging and cytological findings. Lesions identified on subtracted images were categorised according to the Breast Imaging Reporting and Data System (BI-RADS) lexicon [21] based on lesion morphology 
(shape, rim, internal structure) and dynamic contrast enhancement patterns determined from signal intensity (SI)-time curves obtained at regions of interest (ROIs) drawn on breast lesions and areas of normal breast tissue.

Lesions classified as BI-RADS 1, 2, and 3 were considered benign while lesions classified as BI-RADS 4 and 5 were considered malignant. Criteria for classifying lesions as BI-RADS 3 (probably benign) were regular margins, early intense and homogenous enhancement and a type II SI-time curve. All patients with lesions categorized as BI-RADS 1, 2 or 3 were evaluated by means of follow-up at $6,12,18$ and 24 months involving physical examination, ultrasound (and DM in patients $>35$ years of age) and cytological examination of the nipple discharge. Patients with papillary, atypical, suspicious, or malignant cells at cytological examination underwent surgery. Patients with lesions classified as BI-RADS 4 or 5 underwent core needle biopsy under imaging guidance, followed by surgical intervention as appropriate.

The performance of $\mathrm{MRI}$ in positive cases on conventional imaging was also evaluated in comparison of DM and US.

\section{Statistical analysis}

The diagnostic performance of breast MRI for the detection of malignant disease was determined using findings from histology or 2-year follow-up as reference standard. True positive (TP) malignant disease was diagnosed when lesions classified as BI-RADS 4 or 5 at MRI were confirmed as malignant at histology. Conversely, false positive (FP) malignant disease was diagnosed when lesions classified as BI-RADS 4 or 5 at MRI were confirmed as benign at histology. True negative (TN) disease was diagnosed when lesions classified as BI-RADS 1, 2 or 3 at MRI were confirmed as benign at ultrasound and cytological examination after 24 months or with a benign result at histology. Finally, false negative (FN) disease was diagnosed when lesions classified as BI-RADS 1, 2 or 3 at MRI were confirmed as malignant at pathology.

Analysis was also performed to determine whether MRI provides additional diagnostic information in cases of positive findings on conventional imaging (DM and US).

\section{Results}

Based on MRI findings, 32 patients were classi-

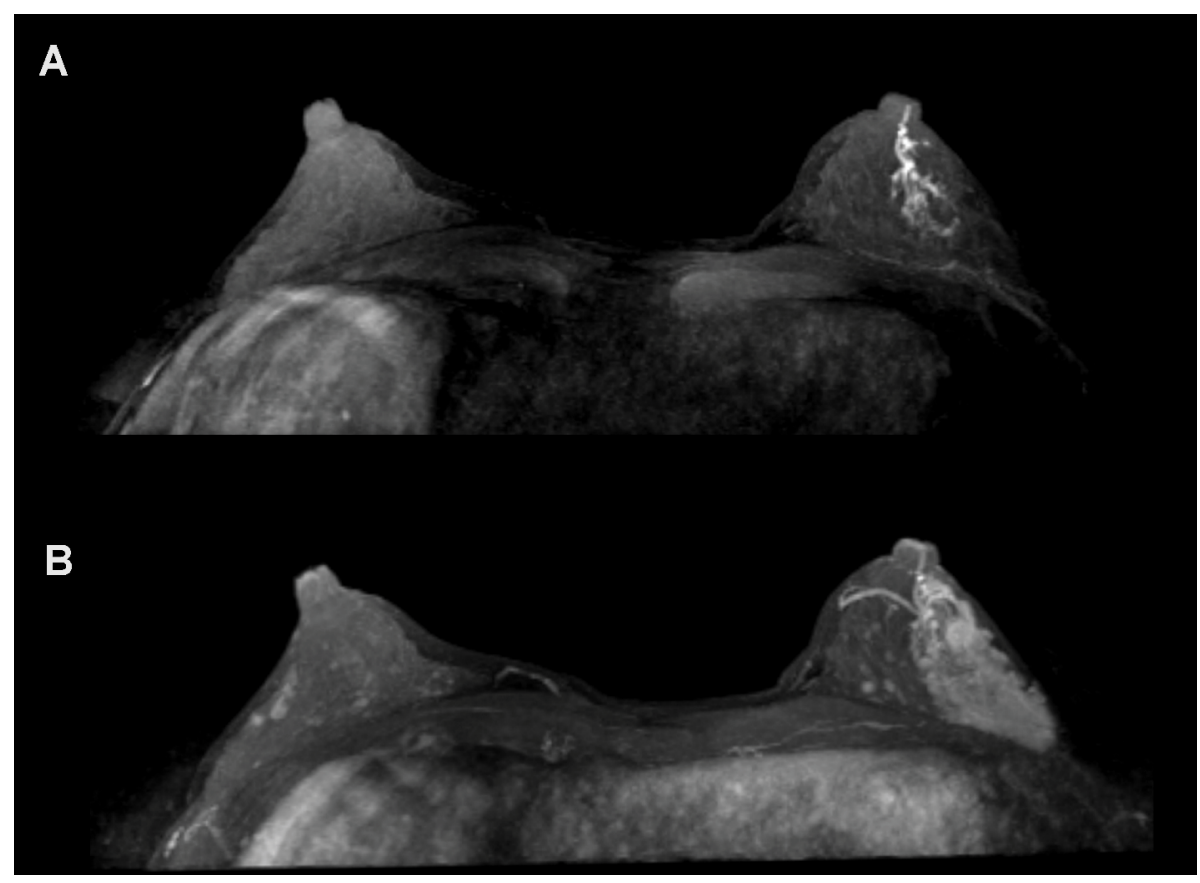

Figure 1: 37-year-old woman with spontaneous and bloody left nipple discharge.

A) The unenhanced T1-weighted MIP reconstruction revealed a hyperintense signal in the ducts suggestive of bloody endoluminal materials. B) The post-contrast T1-weighted MIP reconstruction revealed an extensive non-mass area of segmental enhancement that extended into the outer quadrants up to the retroareolar region. The lesion was classified as MR-BIRADS 4, consistent with carcinoma of the breast, which was confirmed by histopathology as invasive ductal carcinoma. 
fied as BI-RADS 4 or 5 and underwent initial core needle biopsy (Figure 1). Of the remaining 47 patients classified as BI-RADS 1, 2 or 3, 28 patients underwent regular follow-up over 24 months and 19 underwent surgery on the basis of cytological findings or Multi-Disciplinary Team indications.

The 51 lesions detected at histologic evaluation included 29 malignant lesions (IDC, $n=6$; IDC with in situ foci, $n=5$; papillary carcinoma, $n=6$; DCIS, $n=8$; invasive lobular carcinoma, $n=1$; lobular carcinoma in situ with invasive component, $\mathrm{n}=$ 1 ; invasive lobular carcinoma with foci of IDC, $n=$ 1; and DCIS associated with Paget disease, $n=1)$. Other lesions were papillomas ( $n=9$, including 2 intraductal), papillomatosis $(n=3)$, one case of inflammation $(n=1)$, sclerosing adenosis $(n=1)$, intraductal papillary proliferation $(n=1)$, hyperplasia with no atypia $(n=1)$, atypical ductal hyperplasia ( $n$ $=1)$, lobular intrahepitelial hyperplasia (LIN) $(n=1)$, benign phyllodes tumor $(n=1)$, complex sclerosing lesion ( $n=1)$, granulomatous mastitis $(n=1)$ and at least one case of B1 $(n=1)$.

\section{Diagnostic performance of MRI}

Overall, 27 patients classified as BI-RADS 4 or 5 were considered as TP for malignant lesions while 45 patients classified as BI-RADS 1, 2 or 3 were considered as TN. Conversely, 5 patients classified as Bi-RADS 4 or 5 were confirmed as FP for malignant disease while 2 patients classified as BI-RADS 1, 2 or 3 were confirmed as FN. The sensitivity, specificity and accuracy of MRI for the detection of breast cancer in women with suspicious ND were therefore $93.1 \%$ (27/29), 90\% (45/50) and 91.1\% (72/79), respectively, while the PPV and NPV were $84.3 \%$ (27/32) and $95.7 \%$ (45/47), respectively. The five FP patients included four patients classified as BI-RADS 4 who were subsequently diagnosed with inflammation ( $\mathrm{n}$ $=1)$, sclerosing adenosis $(n=1)$, papilloma $(n=1)$ and papillomatosis $(n=1)$ and one patient classified as BI-RADS 5 who was subsequently diagnosed with intraductal papilloma. Conversely, the two FN patients, each classified as BI-RADS 1, were subsequently diagnosed with DCIS and DCIS/Paget disease. One patient with unilateral bloody nipple discharge had negative findings at DM, US and MRI but had atypical findings at cytological examination. This patient underwent surgery which revealed a tiny $(2 \mathrm{~mm})$ papilloma.

\section{Comparison with conventional imaging}

MRI findings were compared with findings from DM and US to determine the possible advantages of MRI in patients with positive US and/or DM. For this evaluation all patients with positive findings at conventional imaging which were classified as BIRADS 4 and $5(n=21)$, as well as patients with US or DM findings classified as BI-RADS $3(n=17)$, were included (Table 1).

Table 1: Characteristics and BIRADS score of the 38 selected patients with ultrasound and digital mammography findings classified as BIRADS 3, 4 or 5. DM microcalc: presence of suspicious microcalcifications on digital mammography; HISTO: histology; $n / a$ : not available.

\begin{tabular}{|c|c|c|c|c|c|c|c|c|c|c|c|}
\hline $\begin{array}{l}\text { MX } \\
\text { BIRADS }\end{array}$ & $\begin{array}{l}\text { US } \\
\text { BIRADS }\end{array}$ & $\begin{array}{l}\text { MRI } \\
\text { BIRADS }\end{array}$ & Histology & $\begin{array}{l}\mathrm{MX} \\
\text { micro }\end{array}$ & $\begin{array}{l}\text { US Size } \\
(\mathrm{mm})\end{array}$ & $\begin{array}{l}\text { MRI } \\
1^{\circ} \\
\text { Lesion } \\
(\mathrm{mm})\end{array}$ & $\begin{array}{l}\text { MRI } \\
2^{\circ} \\
\text { Lesion } \\
(\mathrm{mm})\end{array}$ & $\begin{array}{l}\text { MRI } \\
3^{\circ} \text { Lesion } \\
(\mathrm{mm})\end{array}$ & $\begin{array}{l}\text { Histo } \\
1^{\circ} \text { Lesion } \\
(\mathrm{mm})\end{array}$ & $\begin{array}{l}\text { Histo } \\
2^{\circ} \text { Lesion } \\
(\mathrm{mm})\end{array}$ & $\begin{array}{l}\text { Histo } \\
3^{\circ} \text { Lesion } \\
(\mathrm{mm})\end{array}$ \\
\hline 1 & 3 & 5 & IDC + DCIS & & 7 & 60 & 32 & 7 & 58 & 28 & 7 \\
\hline 4 & 4 & 4 & IDC & & 15 & 50 & & & 50 & & \\
\hline 3 & 1 & 4 & $\begin{array}{l}\text { Intracystic } \\
\text { papillary cancer }\end{array}$ & $x$ & no & 15 & 8 & & 15 & 10 & \\
\hline 4 & 4 & 5 & IDC & $x$ & 25 & 80 & & & 90 & & \\
\hline 1 & 3 & 5 & $\begin{array}{l}\text { Intraductal } \\
\text { papilloma }\end{array}$ & & 7 & 9 & & & 10 & & \\
\hline 5 & 3 & 5 & ID + DCIS & $x$ & 15 & 40 & 18 & 15 & 40 & 20 & 15 \\
\hline 1 & 3 & 4 & $\begin{array}{l}\text { Sclerosing } \\
\text { adenosis }\end{array}$ & & 6 & 10 & & & 10 & & \\
\hline 4 & 3 & 4 & $\begin{array}{l}\text { Papillary } \\
\text { carcinoma }\end{array}$ & $x$ & 13 & 13 & & & 15 & & \\
\hline n/a & 4 & 1 & Follow up & & 19 & 0 & & & & & \\
\hline 1 & 4 & 5 & ILC & & 20 & 30 & & & 28 & & \\
\hline
\end{tabular}




\begin{tabular}{|c|c|c|c|c|c|c|c|c|c|c|c|}
\hline 0 & 4 & 2 & $\begin{array}{l}\text { Usual ductal } \\
\text { hyperplasia }\end{array}$ & & 12 & 13 & & & 13 & & \\
\hline 4 & 1 & 5 & DCIS & $\mathrm{X}$ & No & 25 & 16 & 9 & 22 & 15 & 7 \\
\hline 3 & 3 & 5 & $\begin{array}{l}\text { Papillary } \\
\text { carcinoma }\end{array}$ & & 20 & 25 & & & 24 & & \\
\hline 5 & 5 & 5 & IDC & & 25 & 25 & & & 25 & & \\
\hline 5 & 5 & 5 & IDC + DCIS & $X$ & 40 & 50 & 24 & 9 & 52 & 25 & 10 \\
\hline 0 & 3 & 2 & Follow-up & & 10 & 15 & & & & & \\
\hline$n / a$ & 3 & 2 & Follow-up & & 10 & 13 & & & & & \\
\hline 5 & 5 & 5 & IDC & & 20 & 40 & 22 & & 39 & 20 & \\
\hline 2 & 3 & 3 & Papillomatosis & & 15 & 12 & & & 15 & & \\
\hline 4 & 1 & 5 & DCIS & $X$ & no & 35 & & & 35 & & \\
\hline 3 & 3 & 4 & $\begin{array}{l}\text { Papillary } \\
\text { carcinoma }\end{array}$ & & 25 & 25 & 12 & & 25 & 10 & \\
\hline 4 & 4 & 3 & Follow-up & & 13 & 15 & & & & & \\
\hline $\mathrm{n} / \mathrm{a}$ & 3 & 2 & $\begin{array}{l}\text { Sclerosing } \\
\text { adenosis }\end{array}$ & & 10 & 12 & & & & & \\
\hline 4 & 1 & 3 & Follow-up & $\mathrm{X}$ & no & 8 & & & & & \\
\hline$n / a$ & 4 & 2 & Follow-up & & 18 & 20 & & & & & \\
\hline $\mathrm{n} / \mathrm{a}$ & 3 & 2 & Follow-up & & 15 & 10 & & & & & \\
\hline 5 & 5 & 5 & $\begin{array}{l}\text { Multicentric } \\
\text { IDC }\end{array}$ & & 12 & 35 & 26 & 10 & 30 & 30 & 10 \\
\hline$n / a$ & 4 & 3 & $\begin{array}{l}\text { Benign } \\
\text { phyllodes } \\
\text { tumour }\end{array}$ & & 40 & 40 & & & 43 & & \\
\hline$n / a$ & 4 & 2 & $\begin{array}{l}\text { Granulomatous } \\
\text { mastitis }\end{array}$ & & 25 & 25 & & & 25 & & \\
\hline 4 & 1 & 5 & DCIS & $X$ & 0 & 30 & & & 38 & & \\
\hline 4 & 4 & 3 & $\begin{array}{l}\text { Complex } \\
\text { sclerosing } \\
\text { lesion }\end{array}$ & & 22 & 23 & & & 23 & & \\
\hline 4 & 4 & 5 & IDC + ILC & & 22 & 35 & & & 38 & & \\
\hline $\mathrm{n} / \mathrm{a}$ & 3 & 2 & Follow-up & & 7 & 7 & & & & & \\
\hline 3 & 1 & 3 & Follow-up & & no & 10 & 6 & & & & \\
\hline 3 & 3 & 3 & Papilloma & & 5 & 5 & & & 5 & & \\
\hline$n / a$ & 3 & 4 & IDC & & 15 & 10 & & & 11 & & \\
\hline 0 & 3 & 4 & IDC & & 21 & 45 & 23 & & 45 & 24 & \\
\hline $\mathrm{n} / \mathrm{a}$ & 3 & 4 & Papillomatosis & & 6 & 8 & & & 8 & & \\
\hline
\end{tabular}

Overall $(n=38), 6$ patients $(15.7 \%)$ had a positive DM (considering positive any findings classified as BI-RADS 3, 4 or 5 ) but negative US examination (BI-RADS 1 or 2 ) while 18 patients (47.3\%) had positive US but negative or not available DM examination. Only in 14 cases (36.8\%) were both DM and US positive. In those patients with positive DM and negative US $(n=6), M R I$ revealed at least one enhancing lesion.

Of the 32 patients with positive US findings (Figure 2), a difference in lesion size of $>2 \mathrm{~mm}$ between $\mathrm{MRI}$ and US was reported in 18 patients. Of these, the $83 \%(15 / 18)$ of detected lesions were larger on MRI than on US (including 7 lesions that were at least $50 \%$ larger on MRI; Figure 3) while in $3 / 18(17 \%)$ patients the lesion was smaller on MRI, including 1 in which the MRI examination was negative. The remaining $14 / 32$ patients, the lesion size on US and MRI was equivalent ( $\leq 2 \mathrm{~mm}$ ).

In $10 / 38$ patients (26\%) MRI revealed the presence of one more lesion not detected on conventional imaging: MRI revealed one additional enhancing lesions in 5/10 patients and two additional lesions in the remaining $5 / 10$ patients.

Of the 17 patients with US or DM findings clas- 


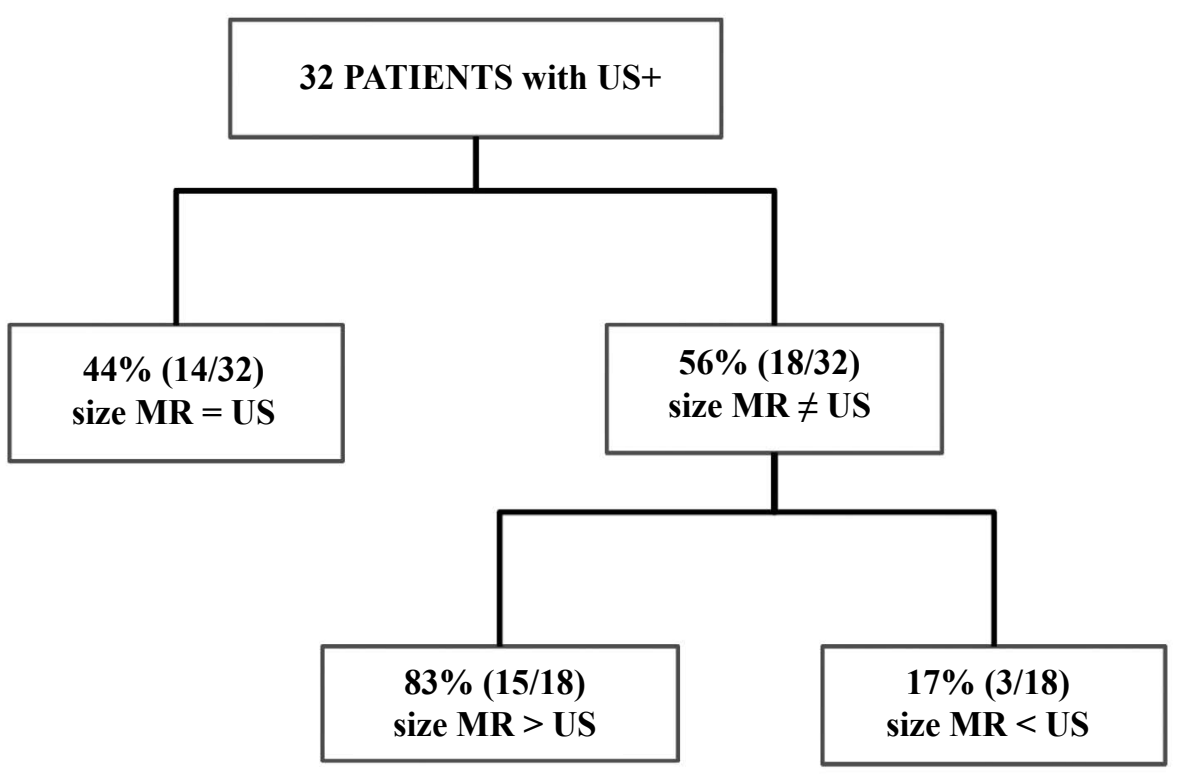

Figure 2: Correlation between Ultrasound and MR measurements of the 32 patients with BIRADS 3,4 or 5 US findings.

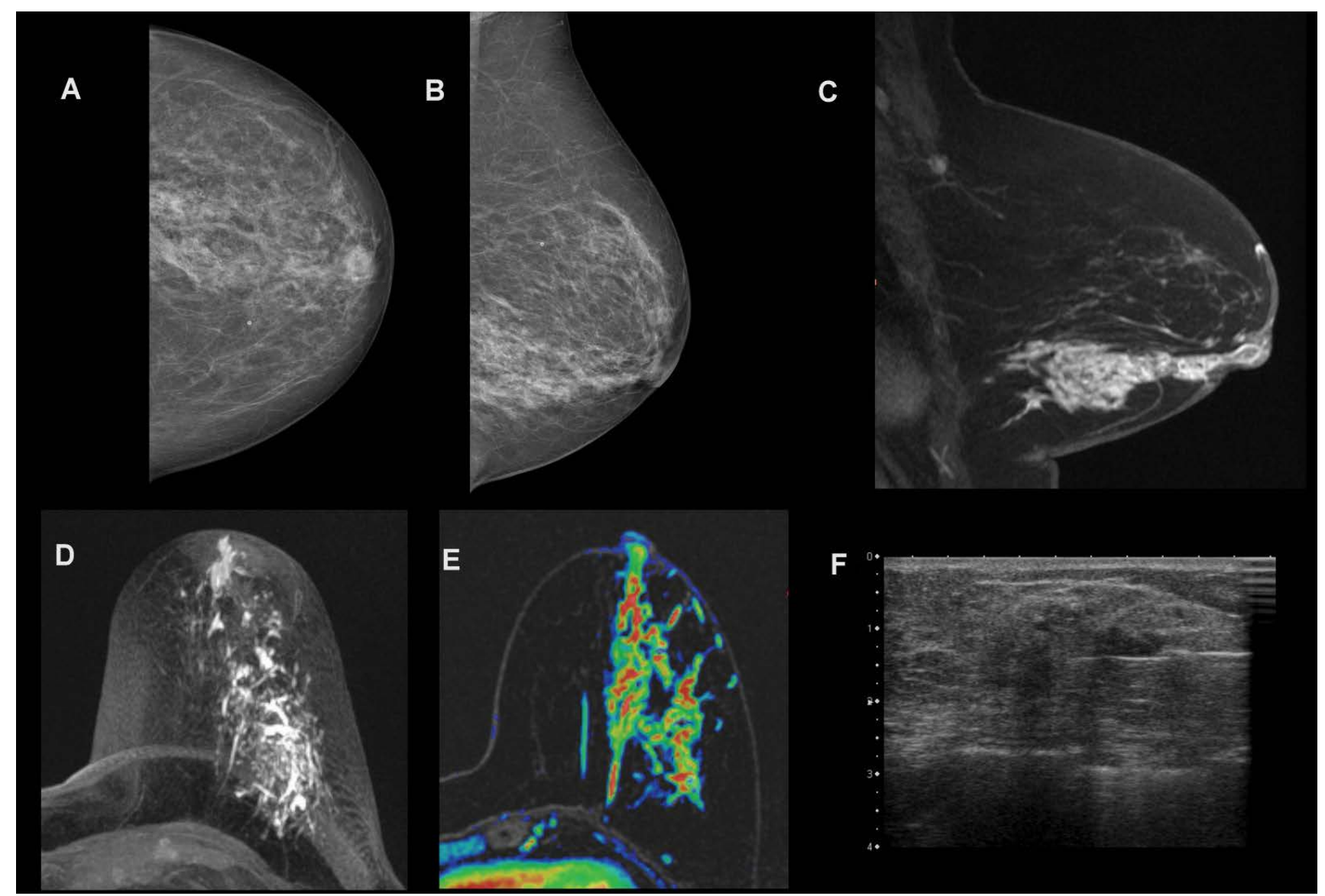

Figure 3: 60-year-old woman with pathologic discharge from left nipple. A, B) Digital mammography (craniocaudal and mediolateral-oblique views) reveals an area of glandular density with some amorphous microcalcifications.

C, E) Contrast-enhanced MR image shows an $8 \mathrm{~cm}$ segmental "non-mass" enhancing area the outer-lower quadrant of left breast. D) The T1-weighted image in the axial plane shows ductal ectasia with endoluminal proteinaceous material. F) Ultrasound showed a $2.5 \mathrm{~cm}$ heterogeneous hypoechoic area on which biopsy was performed. Invasive ductal carcinoma was revealed. 


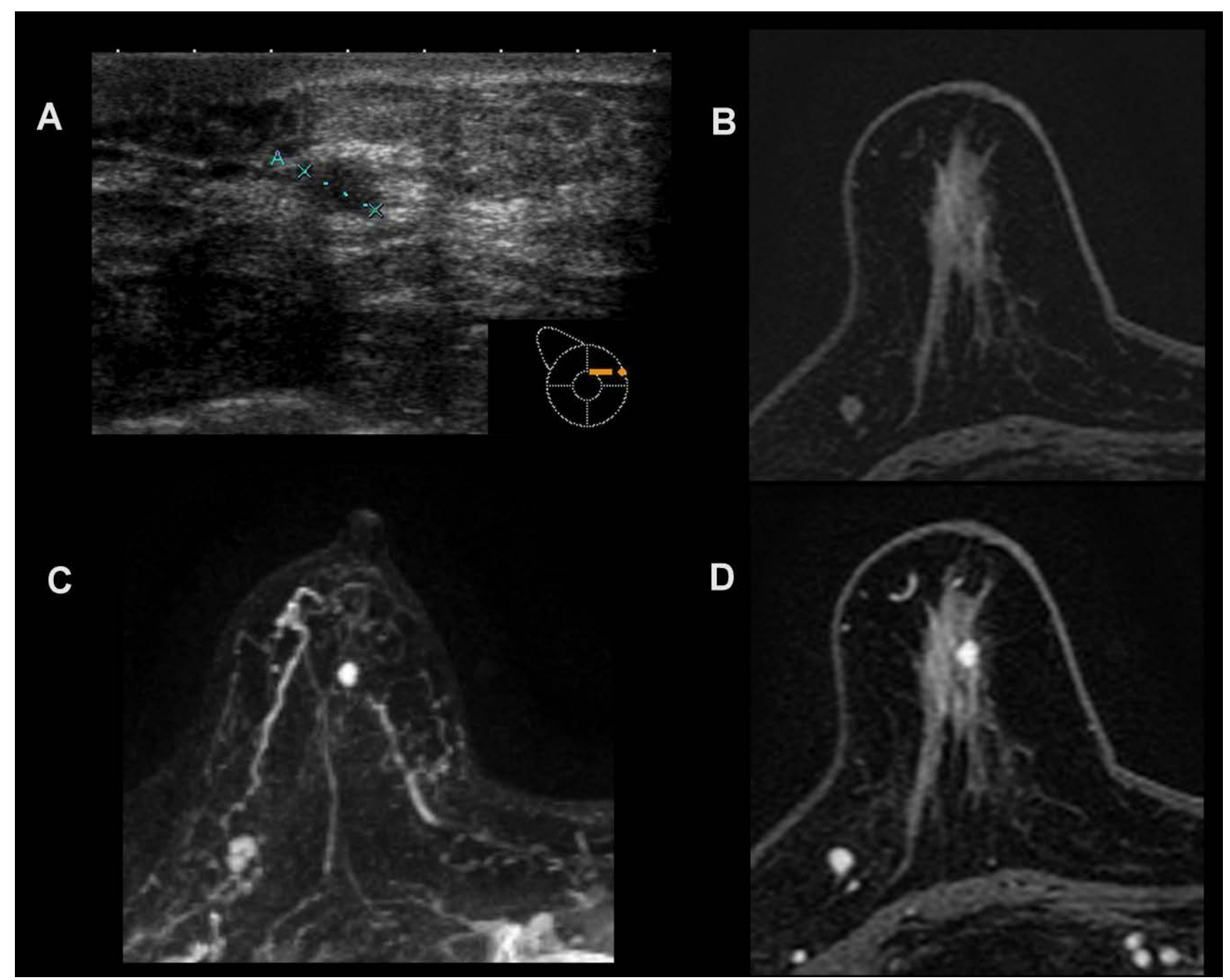

Figure 4: 83-year-old patient with clear-bloody discharge from the right nipple and (A) finding of $5 \mathrm{~mm}$ nodular hypoechoic lesion on US classified as BIRADS 4 which is not identifiable on pre-contrast MRI image (B).

C, D) After contrast administration a $5 \mathrm{~mm}$ single nodular enhancement with regular margin has observed on MRI. No more suspicious enhancements were seen and the finding was classified as BIRADS 3. Multi-Disciplinary-Team decided to perform a core biopsy and diagnosis was papilloma

sified as BI-RADS 3, MRI has given a different BIRADS classification in 14/17 patients, based on the morphology and dynamic contrast enhancement patterns of lesions on MRI. In 9 patients (53\%) the classification was changed from a BI-RADS 3 to MR BI-RADS 4 or 5 (of which 6 lesions were confirmed as malignant at histology) while in 5 (29\%) the BIRADS score of 3 was altered to MR BI-RADS 1 and 2. One of these 5 lesions was confirmed as benign at histology and the remaining 4 at follow-up. In 3 cases (18\%) MR BI-RADS score were in agreement with BI-RADS 3, and all lesions were confirmed as benign by histology or follow-up.

Of the 21 patients with BI-RADS 4 and 5 at conventional imaging, MRI permits to decrease the $\mathrm{BI}$ RADS ratio to BI-RADS 1, 2 or 3 in 8 cases and all of these were confirmed as benign at histology $(n=4)$ or at follow-up $(n=4)$ (Figure 4).

\section{Discussion}

Although PND is mostly caused by papilloma (35-56\%) or benign duct ectasia (6-59\%), it can also indicate an underlying malignancy (5-23\%), typically DCIS or IDC $[9,22-23]$. Although nearly $80 \%$ of women with ND have a benign disease, there is no clear consensus as to what differentiates a benign cause from a malignant cause based on clinical and radiographic assessments [17]. For patients with PND, the standard of care includes mammography and ultrasound, which may not show any pathologic findings. Positive mammography findings have been reported in $50-90 \%$ of patients with malig- 
nancy but in less than $50 \%$ of patients with a benign papilloma [24]. Ultrasound has a sensitivity of $97 \%$, a specificity of $60 \%$, and a PPV of $95 \%$ for the detection of any kind of lesion [25]. Alternative approaches include galactography, ductoscopy or central duct excision; these procedures are invasive and anesthesia could be necessary but, on the other hand, they can offer a direct therapeutic approach [26]. Unfortunately, these techniques, either alone or in combination, have not proven reliable in accurately diagnosing the cause of PND $[8-10,12,15,27,28]$. A newer approach, which is increasingly applied in this clinical setting, is MRI [18].

Whereas MRI has previously been compared with galactography [29], we evaluated the added value of MRI compared with conventional imaging methods (US and DM). To our knowledge, this is the first study to investigate the performance of MRI at 3.0 Tesla in the evaluation of nipple discharge in patients with positive DM and/or US. We noted a higher rate of lesion detection on MRI than on US or DM, and better overall agreement with pathology findings in terms of disease extension.

As noted by Mann, et al. [30] whereas unenhanced MRI can differentiate lesions and abnormalities of the breast, administration of a gadolinium-containing contrast material is needed to accurately diagnose or exclude a cancer. In our study MRI showed high sensitivity $(93.1 \%, 27 / 29)$ for breast lesion detection and diagnosis, supporting numerous previous studies that have confirmed dynamic contrast enhancement MRI as the most sensitive diagnostic approach for breast cancer detection $[7,8,19]$. Our results are consistent with the pooled sensitivity reported in a recent meta-analysis [29]. Our specificity $(90 \%,[45 / 50])$ and PPV (84.3\%, [27/32]) were higher than the values reported previously with the exception of findings reported by Manganaro, et al. [19]. In part the excellent diagnostic performance achieved in our study can be ascribed to the use of a 3.0 Tesla magnet rather than a 1.5 Tesla magnet for which sensitivity and specificity values in patients with ND have ranged from $60-95 \%$ and $44-79 \%$, respectively $[7,10,22,31]$. Improved sensitivity at 3.0 Tesla was also reported by Pinker, et al. [32] in an intra-individual comparison of 3.0 Tesla and 1.5 Tesla MRI; they reported significantly better image quality at 3.0 Tesla due to the higher spatial resolution.

As reported elsewhere [33], we noted a high NPV $(95.7 \%, 45 / 47)$ at MRI in the setting of ND
$[7,8,14,15]$. Notably, the two false-negative malignancies found at MRI were DCIS and DCIS/Paget disease.

In agreement with previous studies $[7,10]$ we noted a considerable difference in the sensitivity of MRI compared with that of US and DM. MRI is known to detect significantly more cancers than US and $\mathrm{DM}$ for lesions $\leq 10 \mathrm{~mm}$ in size or presenting as non-mass enhancement (NME) [8,34].

Of the $34 \%$ of malignant lesions confirmed by histology (10/29), conventional imaging methods did not detect the presence of cancer and among these, 5 patients were subsequently shown to have DCIS and 3 a malignancy with an in situ component. In all of these cases, MRI revealed the presence of linear NME. Linear NME is often responsible for differences in lesion size determined on MRI and US. In 56\% (18/32) of patients MRI revealed a maximum lesion diameter that was at least $3 \mathrm{~mm}$ larger than that revealed on US which can be ascribed to the linear component not being seen at US. Importantly, MRI allowed us to identify not only enhancing lesions in patients with negative US and positive DM but also the presence of occult lesions not visualized on conventional imaging.

Although a correlation between histopathology and MRI was not the goal of our study, we noted good agreement between the dimensions estimated on MRI and those measured on the pathological sample. This is at variance with previous reports in which MRI measurements were frequently overestimated $[35,36]$.

Our study revealed a marked impact of $M R I$ on the assignation of BI-RADS scores. Specifically, MRI altered the BI-RADS classification for $58 \%(22 / 38)$ of patients with BI-RADS scores assigned on the basis of US and/or DM. Lesions classified as BI-RADS 3 on DM or US were considerably reducing (from 17 to 7$)$. In 9/17 cases MRI resulted in a change from BI-RADS score from 3 to 4 or 5 due to the detection of malignant features not revealed at conventional imaging and of these 6 lesions were confirmed to be cancers by the histological diagnosis. Therefore, patients underwent to surgery rather than follow-up. In $38 \%$ of cases, MRI changed BI-RADS classification from 4 or 5 to category 1,2 , or 3 and all of these lesions were confirmed as benign at histology or follow-up.

A more accurate assignation of BI-RADS scores with MRI has a clinically importance in reducing the 
need and costs associated with further invasive examinations such as galactography, ductoscopy or central duct excision. Furthermore, the high sensitivity and NPV associated with a negative breast MRI would clearly influence patient management decisions, potentially resulting in reduced interventions and increased follow-up, thereby minimizing patient anxiety.

A limitation of our study is the relatively small number of patients. A second limitation is that evaluation was performed in consensus by two experienced readers that were blinded to all patient-related information as well as to the results of conventional imaging. In routine practice readers would have access to all available information during image evaluation.

In conclusion, MRI proved the most sensitive and diagnostically accurate test for the evaluation of PND. In our opinion MRI should not only be preferred over galactography for further patient workup if DM and US are negative or inconclusive but should also be performed in patients with positive findings on conventional imaging because of its greater sensitivity for lesion detection.

\section{References}

1. Leis HP Jr (1989) Management of nipple discharge. World J Surg 13: 736-742.

2. Stone K, Wheeler A (2015) A review of anatomy, physiology, and benign pathology of the nipple. Ann Surg Oncol 22: 3236-3240.

3. Patel BK, Falcon S, Drukteinis J (2015) Management of nipple discharge and the associated imaging findings. Am J Med 128: 353-360.

4. Okuda Y, Kodama $Y$ (2012) Suspicious nipple discharge diagnostic evaluation. Dr. Laszlo Tabar, Imaging of the breast - technical aspects and clinical implication.

5. Lanitis S, Filippakis G, Thomas J, Christofides T, Al Mufti R, et al. (2008) Microdochectomy for single-duct pathologic nipple discharge and normal or benign imaging and cytology. Breast 17: 309-313.

6. Orel SG, Dougherty CS, Reynolds C, Czerniecki BJ, Siegelman ES, et al. (2000) MR imaging in patients with nipple discharge: Initial experience. Radiology 216: 248-254.

7. Lorenzon M, Zuiani C, Linda A, Londero V, Girometti $R$, et al. (2011) Magnetic resonance imaging in patients with nipple discharge: Should we recommend it? Eur Radiol 21: 899-907.
8. Lubina $N$, Schedelbeck $U$, Roth $A$, Weng AM, Geissinger $E$, et al. (2015) 3.0 Tesla breast magnetic resonance imaging in patients with nipple discharge when mammography and ultrasound fail. Eur Radiol 25: 1285-1293.

9. Lippa N, Hurtevent-Labrot G, Ferron S, Boisserie Lacroix M (2015) Nipple discharge: The role of imaging. Diagn Interv Imaging 96: 1017-1032.

10. Morrogh M, Park A, Elkin EB, King TA (2010) Lessons learned from 416 cases of nipple discharge of the breast. Am J Surg 200: 73-80.

11.Lau S, Küchenmeister I, Stachs A, Gerber B, Krause A, et al. (2005) Pathologic nipple discharge: Surgery is imperative in postmenopausal women. Ann Surg Oncol 12: 546-551.

12. Simmons R, Adamovich $T$, Brennan $M$, Christos $P$, Schultz M, et al. (2003) Nonsurgical evaluation of pathologic nipple discharge. Ann Surg Oncol 10: 113-116.

13.Dupont SC, Boughey JC, Jimenez RE, Hoskin TL, Hieken TJ (2015) Frequency of diagnosis of cancer or high-risk lesion at operation for pathologic nipple discharge. Surgery 158: 988-994.

14. Nakahara H, Namba K, Watanabe R, Furusawa $\mathrm{H}$, Matsu T, et al. (2003) A comparison of MR imaging, galactography and ultrasonography in patients with nipple discharge. Breast Cancer 10: 320-329.

15.Ballesio L, Maggi C, Savelli S, Angeletti M, De Felice C, et al. (2008) Role of breast magnetic resonance imaging (MRI) in patients with unilateral nipple discharge: Preliminary study. Radiol Med 113: 249-264.

16. Mortellaro VE, Marshall J, Harms SE, Hochwald SN, Copeland EM, et al. (2008) Breast MR for the evaluation of occult nipple discharge. Am Surg 74: 739-742.

17.Nicholson BT, Harvey JA, Patrie JT, Mugler JP (2015) 3D-MR ductography and contrast-enhanced MR mammography in patients with suspicious nipple discharge; a Feasibility Study. Breast J 21: 352-362.

18. Bahl M, Baker JA, Greenup RA, Ghate SV (2015) Evaluation of pathologic nipple discharge: What is the added diagnostic value of MRI? Ann Surg Oncol 22: 435-441.

19. Manganaro L, D'Ambrosio I, Gigli S, Di Pastena F, Giraldi $G$, et al. (2015) Breast MRI in patients with unilateral bloody and serous-bloody nipple discharge: A comparison with galactography. Biomed Res Int 2015: 806368.

20.Sanders LM, Daigle M (2016) The rightful role of MRI after negative conventional imaging in the management of bloody nipple discharge. Breast J 22: 209212. 
21.Morris EA, Comstock CE, Lee CH (2013) ACR BI-RADS ${ }^{\circledR}$ Magnetic Resonance Imaging. In: ACR BI-RADS ${ }^{\circledast}$ Atlas, Breast Imaging Reporting and Data System. Reston, VA, American College of Radiology.

22.Morrogh $\mathrm{M}$, Morris EA, Liberman L, Borgen PI, King TA (2007) The predictive value of ductography and magnetic resonance imaging in the management of nipple discharge. Ann Surg Oncol 14: 3369-3377.

23.Van Gelder L, Bisschops RH, Menke-Pluymers MB, Westenend PJ, Plaisier PW (2015) Magnetic resonance imaging in patients with unilateral bloody nipple discharge; useful when conventional diagnostics are negative? World J Surg 39: 184-186.

24. Hirose M, Nobusawa H, Gokan T (2007) MR ductography: Comparison with conventional ductography as a diagnostic method in patients with nipple discharge. Radio Graphics 27: S183-S196.

25.Ballesio L, Maggi C, Savelli S, Angeletti M, Rabuffi P, et al. (2007) Adjunctive diagnostic value of ultrasonography evaluation in patients with suspected ductal breast disease. Radiol Med 112: 354-365.

26.Dietz JR, Crowe JP, Grundfest S, Arrigain S, Kim JA (2002) Directed duct excision by using mammary ductoscopy in patients with pathologic nipple discharge. Surgery 132: 582-588.

27.Jeffrey P Lamont, Rachel P Dultz, Joseph A Kuhn, Michael D Grant, Ronald C Jones (2000) Galactography in patients with nipple discharge. Proc (Bayl Univ Med Cent) 13: 214-216.

28.Cho N, Moon WK, Chung SY, Cha JH, Cho KS, et al. (2005) Ductographic findings of breast cancer. Korean J Radiol 6: 31-36.
29. Berger N, Luparia A, Di Leo G, Carbonaro LA, Trimboli RM, et al. (2017) Diagnostic performance of MRI versus galactography in women with pathologic nipple discharge: A systematic review and meta-analysis. AJR 209: 465-471.

30.Mann RM, Balleyguier C, Baltzer PA, Bick U, Colin C, et al. (2015) Breast MRI: EUSOBI recommendations for women's information. Eur Radiol 25: 3669-3678.

31.Albrecht C, Thele F, Grunwald S, Kohlmann T, Hegenscheid K, et al. (2013) Nipple discharge: Role of ductoscopy in comparison with standard diagnostic tests. Onkologie 36: 12-16.

32.Pinker K, Grabner G, Bogner W, Gruber S, Szomolanyi $P$, et al. (2009) A combined high temporal and high spatial resolution 3 Tesla MR imaging protocol for the assessment of breast lesions: initial results. Invest Radiol 44: 553-558.

33.Bahl M, Gadd MA, Lehman CD (2017) Journal club: Diagnostic utility of MRI after negative or inconclusive mammography for the evaluation of pathologic nipple discharge. AJR 209: 1404-1410.

34.De Paula IB, Campos AM (2017) Breast imaging in patients with nipple discharge. Radiol Bras 50: 383-388.

35.Son EJ, Kim EK, Kim JA, Kwak JY, Jeong J (2009) Diagnostic value of 3D fast low-angle shot dynamic MRI of breast papillomas. Yonsei Med J 50: 838-844.

36.Schouten van der Velden AP, Schlooz-Vries MS, Boetes C, Wobbes T (2009) Magnetic resonance imaging of ductal carcinoma in situ: What is its clinical application? A review. Am J Surg 198: 262-269. 